INPLASY PROTOCOL

To cite: Ye et al. Efficacy and safety of moxibustion in the treatment of infertility with polycystic ovary syndrome: a protocol of systematic review and meta-analysis. Inplasy protocol 202110004. doi: 10.37766/inplasy2021.1.0004

Received: 01 January 2021

Published: 01 January 2021

Corresponding author: Wenguo Ye

648957697@qq.com

Author Affiliation: Affiliated Hospital of Jiangxi University of Traditional Chinese Medicine

Support: Jiangxi Province.

Review Stage at time of this submission: The review has not yet started.

Conflicts of interest: None.

\section{Efficacy and safety of moxibustion in the treatment of infertility with polycystic ovary syndrome: a protocol of systematic review and} meta-analysis

Ye, WG1; Deng, G2; Yin, L3; Ye, J4.

Review question / Objective: How about the efficacy and safety of moxibustion in the treatment of infertility with polycystic ovary syndrome.

Condition being studied: Polycystic ovary syndrome is one of the common diseases of reproductive endocrine metabolism in gynecology, and it is also a common and difficult disease affecting female reproductive endocrine health. PCOS characterized by insulin resistance and hyperandrogenemia, the clinical manifestations are polychaemia, acne, obesity, infertility, menstrual disorders and so on. PCOS is one of the most important causes of anovulatory infertility. PCOS infertility accounts for $25 \% \sim 30 \%$ of anovulatory infertility. Clinical treatment of patients with PCOS ovulatory dysfunction infertility is mainly treated with ovulationpromoting drugs, insulin sensitizer, hyperandrogenemia drugs and other drugs Healing. It is found that the sensitivity of patients to ovulation promotion is poor, and it is often necessary to increase the dosage of drugs to increase ovulation rate, thus increasing the risk of ovarian hyperstimulation syndrome, and the recurrence rate is higher after withdrawal. Due to the long time, high price, many adverse reactions, protruding side effects, low pregnancy rate, poor curative effect and drug resistance, it has become a difficult problem to be solved in clinic.

INPLASY registration number: This protocol was registered with the International Platform of Registered Systematic Review and Meta-Analysis Protocols (INPLASY) on 01 January 2021 and was last updated on 01 January 2021 (registration number INPLASY202110004).

\section{INTRODUCTION}

Review question / Objective: How about the efficacy and safety of moxibustion in the treatment of infertility with polycystic ovary syndrome.

Rationale: A systematic review and metaanalysis of clinical randomized controlled 
studies on moxibustion in the treatment of infertility with polycystic ovary syndrome following the rules of evidence-based medicine.

Condition being studied: Polycystic ovary syndrome is one of the common diseases of reproductive endocrine metabolism in gynecology, and it is also a common and difficult disease affecting female reproductive endocrine health. PCOS characterized by insulin resistance and hyperandrogenemia, the clinical manifestations are polychaemia, acne, obesity, infertility, menstrual disorders and so on. PCOS is one of the most important causes of anovulatory infertility. PCOS infertility accounts for $25 \% \sim 30 \%$ of anovulatory infertility. Clinical treatment of patients with PCOS ovulatory dysfunction infertility is mainly treated with ovulationpromoting drugs, insulin sensitizer, hyperandrogenemia drugs and other drugs Healing. It is found that the sensitivity of patients to ovulation promotion is poor, and it is often necessary to increase the dosage of drugs to increase ovulation rate, thus increasing the risk of ovarian hyperstimulation syndrome, and the recurrence rate is higher after withdrawal. Due to the long time, high price, many adverse reactions, protruding side effects, low pregnancy rate, poor curative effect and drug resistance, it has become a difficult problem to be solved in clinic.

\section{METHODS}

Search strategy: We will search Eight electronic databases, including PubMed, Embase, Web of Science, Cochrane Library, the China National Knowledge Infrastructure(CNKI), Chinese Science and Technology Periodical Database(VIP), Wanfang Database(WF), and Chinese Biomedical Literature Database(CBM). We will search above electronic databases from the beginning to January 2021, without any language restriction.

Participant or population: There are clear and recognized diagnostic criteria and efficacy criteria, and all patients are diagnosed as infertility with polycystic ovary syndrome, regardless of gender, age and origin of the case.

Intervention: Moxibustion therapy, or mixed therapies based on moxibustion therapy will also be include.

Comparator: The control group will receive one of the following treatment methods: conventional pharma-cological therapy, no treatment, and placebo.

Study designs to be included: Clinical randomized controlled trials (RCTs) containing moxibustion therapy for infertility with polycystic ovary syndrome were included, with no limitation of language and publication status.

Eligibility criteria: Reported in chinese and English, and meet the "PICOS", will be considered for inclusion in this overview.

Information sources: Eight electronic databases will be searched, including PubMed, Embase, Web of Science, Cochrane Library, the China National Knowledge Infrastructure(CNKI), Chinese Science and Technology Periodical Database(VIP), Wanfang Database(WF), and Chinese Biomedical Literature Database(CBM). We will search above electronic databases from the beginning to January 2021, without any language restriction.

Main outcome(s): Ovulation rate and pregnancy rate will be accepted as the primary outcomes.

Additional outcome(s): The changes of Sex hormone levels, including Luteinizing hormone, follicle-stimulating hormone, serum estradiol, total testosterone will be used as secondary outcomes.

Data management: The two researchers independently read the title and abstract of the literature we obtained, read the full text of the trials that might meet the inclusion criteria to determine whether the inclusion criteria were truly met, and discussed the conflicting literatures or let the third researcher decide whether to include them. 
Two researchers independently extracted data from the included studies, including study design, intervention measures and methods, measurement indicators, results, methodological contents such as hidden grouping and blind method, etc., and a third evaluator checked the consistency of the data. If the required information is incomplete, we will contact the original author for the required data.

Quality assessment / Risk of bias analysis: Two evaluators independently select the literature according to the inclusion and exclusion criteria and cross-check. In case of disagreement, a third evaluator will assist in the decision. The extracted data included the first author, year of publication, number of patients, age, gender, intervention measures, outcome indicators, etc. The Jadad scale to evaluate quality into literature, including: random sequence (right 2 points, 1 points not clear, inappropriate 0 ), distribution, hidden (right 2 points, 1 points not clear, inappropriate 0 ), blinded (right 2 points, 1 points not clear, inappropriate 0 ), lost to follow-up and exit (describe 1 points, not describe 0 ); $0-3$ is classified as low quality and 4-7 as high quality.

Strategy of data synthesis: Meta analysis will be performed using Rev Man5.3 software. The odds ratio (OR) and its $95 \%$ Confidence Interval ( $\mathrm{Cl}$ ) will be used as the counting data, while the weighted mean difference (WMD) and its $95 \% \mathrm{Cl}$ will be used as the measurement data.

Subgroup analysis: If heterogeneity exists in the meta-analysis, the source of heterogeneity should be sought, such as whether the degree of disease, treatment cycle, treatment time of each moxibustion, type of intervention, etc., is the source of heterogeneity. If so, a subgroup analysis should be conducted for these reasons to see whether heterogeneity still exists after analysis.

Sensibility analysis: Sensitivity analysis can not only assess the stability and reliability of the combined results, but also assess whether the combined results are significantly changed by the influence of a single study. If sufficient literature is included, we will adopt the method of excluding literature one by one, excluding each included study one by one before effect-size combination, changing the inclusion and exclusion criteria or excluding certain types of literature before effect-size combination.

Language: No limitation of language.

Country(ies) involved: China.

Keywords: Polycystic ovary syndrome; PCOS; protocol; moxibustion; Infertility; systematic review and meta-analysis

Contributions of each author:

Author 1 - Wenguo Ye - The author drafted the manuscript.

Email: 648957697@qq.com

Author 2 - Gen Deng - The author provided statistical expertise.

Email: 1084053154@qq.com

Author 3 - Lin Yin - The author contributed to the development of the selection criteria, and the risk of bias assessment strategy.

Email: 490446887@qq.com

Author 4 - Jing Ye - The author read, provided feedback and approved the final manuscript.

Email: yejing1016@163.com 